\title{
Atomic-Resolution 3D Electron Microscopy with Dynamic Diffraction
}

\author{
Michael A. O’Keefe*, Kenneth H. Downing**, Hans-Rudolf Wenk*** and Hu Meisheng*** \\ *Materials Sciences Division, LBNL 2-200, 1 Cyclotron Road, Berkeley, CA 94720, USA \\ **Life Sciences Division, LBNL Donner, 1 Cyclotron Road, Berkeley, CA 94720, USA \\ ***Department of Geology and Geophysics, University of California, Berkeley, CA 94720.
}

Achievement of atomic-resolution electron-beam tomography will allow determination of the threedimensional structure of nanoparticles (and other suitable specimens) at atomic resolution. Threedimensional reconstructions will yield "section" images that resolve atoms overlapped in normal electron microscope images (projections), resolving lighter atoms such as oxygen in the presence of heavier atoms, and atoms that lie on non-lattice sites such as those in non-periodic defect structures.

Lower-resolution electron microscope tomography has been used to produce reconstructed 3D images of nanoparticles [1] but extension to atomic resolution is considered not to be straightforward. Accurate three-dimensional reconstruction from two-dimensional projections generally requires that intensity in the series of 2-D images be a monotonic function of the specimen structure (often specimen density, but in our case atomic potential). This condition is not satisfied in electron microscopy when specimens with strong periodicity are tilted close to zone-axis orientation and produce "anomalous" image contrast because of strong dynamic diffraction components. Atomicresolution reconstructions from tilt series containing zone-axis images (with their contrast enhanced by strong dynamical scattering) can be distorted when the stronger zone-axis images overwhelm images obtained in other "random" orientations in which atoms do not line up in neat columns.

The first demonstrations of 3-D reconstruction to atomic resolution used five zone-axis images from test specimens of staurolite consisting of a mix of light and heavy atoms [2,3]. Initial resolution was to the $1.6 \AA$ Scherzer limit of a JEOL-ARM1000. Later experiments used focal-series reconstruction from 5 to 10 images to produce staurolite images from the ARM1000 with resolution extended beyond the Scherzer limit to $1.38 \AA$ (fig.1) [4,5]. To obtain a representation of the three-dimensional structure, images were obtained in zone-axis projections $\langle 100\rangle,<010\rangle,<001>,<101\rangle,<310\rangle$, and combined to produce a three-dimensional map of Coulomb potential (fig.2). Images of specimen sections are much more easily interpreted than projection images such as electron micrographs, reducing the need for techniques such as imaging at sub-Rayleigh resolution [6]. Sections through the 3D staurolite potential show atom positions as density peaks (fig.3) that display streaking from insufficient sampling in direction [1]. Three different specimens of perfect crystal were required to achieve the five projection directions; this makes the technique atomic-resolution electron crystallography rather than atomic-resolution tomography. Nevertheless, our results illustrate that dynamic diffraction need not be a limiting factor in atomic-resolution tomographic reconstruction.

We have proposed combining ultra-high (sub-Ångström) resolution zone-axis images with off-zone images by first using linear reconstruction of the off-zone images while excluding images obtained within a small range of tilts (of the order of 60 milliradian) of any zone-axis orientation [7], since it has been shown that dynamical effects can be mitigated by slight off-axis tilt of the specimen [8]. The (partial) reconstruction would then be used as a model for forward calculation by image simulation [9] in zone-axis directions and the structure refined iteratively to achieve satisfactory fits with the experimental zone-axis data. Another path to atomic-resolution tomography would combine "zone-axis tomography" with high-resolution dark-field hollow-cone (DFHC) imaging. Electron diffraction theory indicates that dynamic (multiple) scattering is much reduced under highly- 
convergent illumination. DFHC TEM is the analog of HAADF STEM, and imaging theory shows that image resolution can be enhanced under these conditions [10]. Images obtained in this mode could provide the initial reconstruction, with zone-axis images used for refinement [11].

\section{References}

[1] P.A. Midgley and M. Weyland, Ultramicroscopy 96 (2003) 413-431.

[2] Kenneth H. Downing et al., Nature 348 (1990) 525-528.

[3] Kenneth H. Downing et al. in 49th Ann Proc. EMSA, San Jose, California (1991) 540-541.

[4] H.-R. Wenk, K.H. Downing, Hu Meisheng \& M.A. O'Keefe, Acta Cryst. A48 (1992) 700-716.

[5] M.A. O'Keefe et al., Mat. Res. Soc. Symp. Proc. 332 (1994) 563-571

[6] M.A. O'Keefe, L.F. Allard \& D.A. Blom (2005), these proceedings.

[7] M.A. O'Keefe, L.F. Allard \& K.H. Downing. CNMS Workshop on Microscopy, Metrology \& Manipulations using Electrons, Ions, and Photons. Oak Ridge, Tennessee, September 15-16 (2004).

[8] M.A. O'Keefe \& V. Radmilovic, in 51st Ann Proc. MSA, Cincinnati, Ohio (1993) 980-981.

[9] M.A. O'Keefe, P.R. Buseck and S. Iijima, Nature 274 (1978) 322-324.

[10] M.A. O'Keefe \& A.J. Pitt, Proc. 7th Euro. Cong. on Electr. Micros. Hague, Netherlands (1980) 122-123.

[11] Supported by the Director, Office of Science, Office of Basic Energy Sciences, Materials Sciences and Engineering Division, DOE under contract DE-AC03-76SF00098.

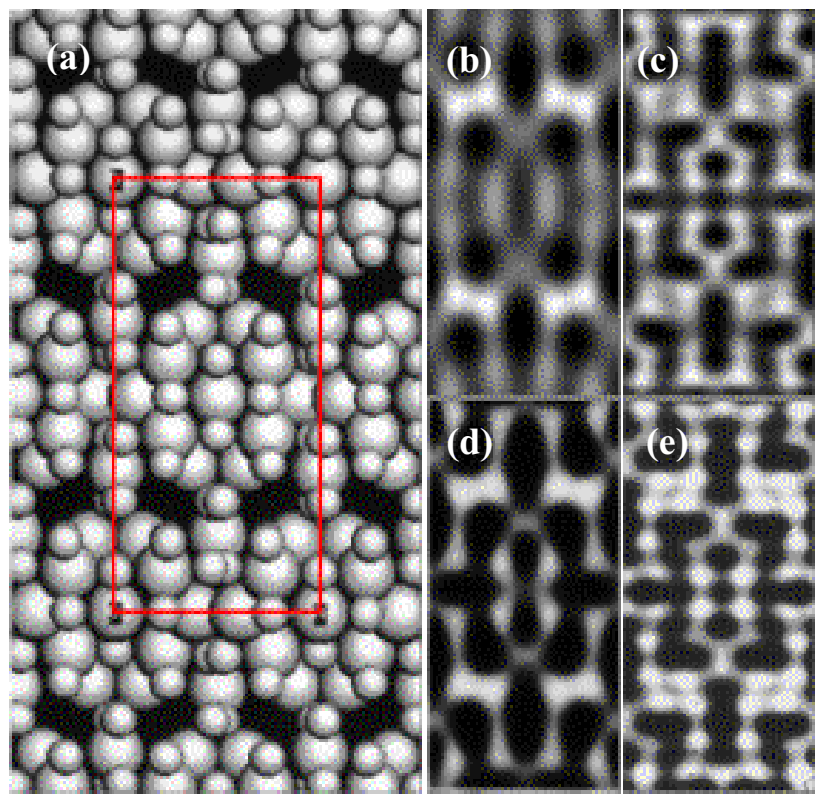

Fig.1. (a) Model of staurolite in [001] direction, with one unit cell marked $(7.82 \AA \times 16.52 \AA)$. Large spheres represent metal atoms and small ones oxygen. (b) Noise-filtered Scherzer image at $1.6 \AA$ resolution. (c) Reconstruction from 10-image focal series yields $1.38 \AA$ resolution. (d, e) Simulations at resolutions of $1.6 \AA$ and $1.38 \AA$ respectively.

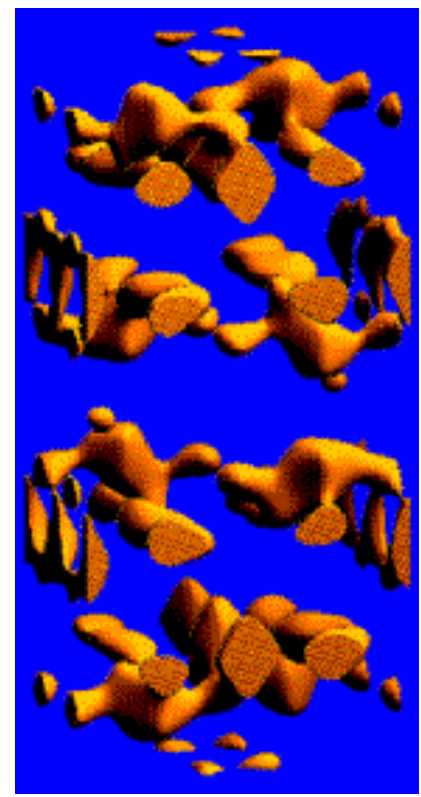

Fig.2. Three-dimensional Coulomb potential in unit cell of staurolite shows all fully occupied cations and oxygen atoms. Reconstructed from five zone-axis images at $1.6 \AA$ resolution.

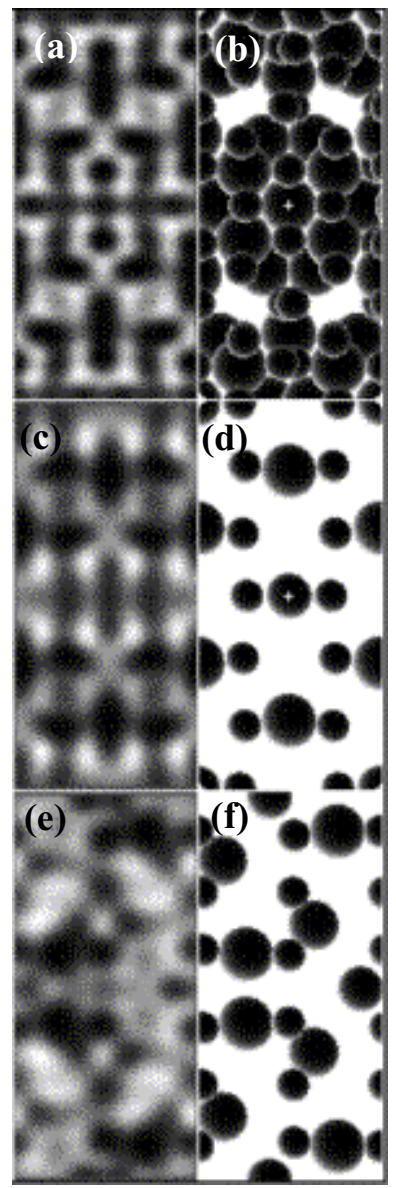

Fig.3. Individual atom positions are clearer in sections than in projection. At $1.38 \AA$ resolution in [001] orientation, experimental image (a) and projected model (b) show overlapping atoms from four layers (spaced $1.4 \AA$ along the $\mathrm{c}$ axis) making up the unit cell. A section through the experimental $3 \mathrm{D}$ reconstruction at $\mathrm{z}=0$ (c) shows peaks at the positions of atoms in the symmetric layer of the model (d). Asymmetrical experimental section at $\mathrm{z}=\mathrm{c} / 4$ (e) also shows peaks at the positions of the atoms making up this layer in the model (f). 